$\begin{array}{cl}\begin{array}{c}\text { Revue } \\ \text { de Ihistoire } \\ \text { des religions }\end{array} & \text { Revue de l'histoire des religions } \\ 1 \mid 2009 \\ \text { Réforme et poésie en Europe aux XVle et XVIle siècles }\end{array}$

\title{
La lyre protestante: Calvin et la réforme poétique en France
}

Protestant poetry: Calvin and the poetical reformation in France

\section{Véronique Ferrer}

\section{OpenEdition}

12 Journals

Édition électronique

URL : http://journals.openedition.org/rhr/7163

DOI : $10.4000 /$ rhr.7163

ISSN : 2105-2573

Éditeur

Armand Colin

Édition imprimée

Date de publication : 1 janvier 2009

Pagination : 55-75

ISBN : 978-2200-92589-5

ISSN : 0035-1423

Référence électronique

Véronique Ferrer, "La lyre protestante : Calvin et la réforme poétique en France », Revue de l'histoire des religions [En ligne], 1 | 2009, mis en ligne le 01 janvier 2012, consulté le 03 mai 2019. URL : http:// journals.openedition.org/rhr/7163; DOI : 10.4000/rhr.7163 


\section{La lyre protestante : Calvin et la réforme poétique en France}

Si la Réforme calvinienne eut les répercussions politiques, sociales et culturelles que l'on sait sur la France du Xvie siècle, elle marqua de manière moins connue mais tout aussi décisive la littérature de son temps. Dans ses Commentaires sur les Psaumes, Calvin mena, toujours sous l'autorité du théologien qu'il fut, une réflexion poétique qui fraya les voies à une poésie originale, éprise d'authenticité et de spiritualité. Nous nous proposons dans cet article de décrypter les grandes lignes de cette poétique réformée et d'en mesurer la portée à l'aune des productions ultérieures des poètes.

\section{Protestant poetry : Calvin and the poetical reformation in France}

The calvinian Reformation had a great influence on French literature during the $16^{\text {th }}$ century, mostly on poetry which it contributed to revive. Through his theological commentaries, Calvin reflected very precisely on biblical style and on the possibility to reform poetry by imitating David's Psalms. In a discontinuous way, his reflection formed a kind of poetical manifesto, which became a reference for French protestant poets. This article aims at drawing the main points of this Protestant poetics, and at examining it in the light of religious lyric poetry at the end of the century. 
La réforme religieuse que mena Calvin était trop exclusive pour faire l'économie d'une révision stylistique. Une spiritualité nouvelle exigeait une écriture ajustée à ses exigences propres. Point de traités théoriques cependant qui consignent les normes d'un langage spécifique, apte à réguler le flux d'une parole littéraire aussi féconde que désorbitée en cette période de renaissances en tous genres. Point de manifestes poétiques non plus qui posent les principes d'une langue et d'une inspiration à proportion des critères de la nouvelle doctrine. Cette vacance théorique ne doit pas être interprétée comme une indifférence pour la poésie ni comme une méfiance à son égard. Bien au contraire. Calvin a luimême composé, dans le secret de son cabinet, des vers laissés en souffrance faute de talent avéré : "Par nature j'étais assez porté à la poésie, écrit-il en 1557 à Conrad Hubert, mais je lui ai dit adieu, et depuis vingt-cinq ans je n'ai rien composé, si ce n'est à Worms, à l'exemple de Philippe [Melanchthon] et de Sturme, je fus amené à écrire par amusement ce poème que tu as $l u »^{1}$. À dire vrai, il reprendra la plume des années plus tard pour traduire quelques psaumes, avant la rédaction du Psautier huguenot par Clément Marot et Théodore de Bèze, puis s'inclinera définitivement devant la supériorité de son premier associé ${ }^{2}$. Ce goût profond

1. Lettre citée par Max Engammare, « Plaisir des mots, plaisir des mets. Irdische Freude bei Calvin », Calvinus Sincerioris Religionis vindex [Proceedings of the international congress for Calvin Research in Edimburgh 1994], ed. by Brian G. Armstrong and Wilhelm H. Neuser, Sixteenth Century Essays and Studies 36, Kirksville, 1997, p. 200-201. Voir aussi «D'une philologie l'autre. La muse classique, maîtresse cachée des Réformateurs », La Philologie humaniste et ses représentations dans la théorie et la fiction, dir. Perrine Galand-Hallyn, Fernand Hallyn et Gilbert Tournoy, Romanica Gadensia n ${ }^{\circ} 32,2005$, p. 409-437, où Max Engammare montre plus généralement l'intérêt très vif que portèrent les Réformateurs à la poésie.

2. Ces traductions de psaumes sont parues dans Aulcuns pseaumes et cantiques mis en chant en 1539. Selon Michel Jeanneret (Poésie et Tradition biblique au XVI siècle, Paris, José Corti, 1969, p. 28-29), les psaumes 25, 46, 36, 91 et 138 sont très vraisemblablement de Calvin. Il cite un extrait de la lettre qu'adressa Calvin à Guillaume Farel lui demandant d'en éprouver la valeur, preuve que ses velléités poétiques demeuraient alors entières : "Nous vous avions envoyé des psaumes pour qu'ils soient chantés chez vous avant d'arriver là où tu sais. Nous avons décidé, en effet de les publier sous peu. Du moment que la mélodie allemande me souriait davantage, il m'a bien fallu éprouver ce que je valais en poésie. Ainsi, les psaumes 46 et 25 sont-ils mes premières recrues; j'en ai composé d'autres dans la suite. » 
pour la poésie explique très certainement l'attention particulière que porte Calvin au style des livres bibliques qu'il commente. Le théologien se sent la vocation d'un poéticien au point d'élaborer de véritables fragments théoriques sur l'art rhétorique, ou sur la langue poétique quand il s'attache aux psaumes. Ses commentaires stylistiques sont autant de fragments d'une poétique idéale, en conformité avec les valeurs spirituelles et morales de la nouvelle religion. En l'absence d'un traité intégral, à nous donc de recomposer une "Défense et illustration de la poésie réformée » à la lumière des métadiscours qui émaillent les Commentaires sur les Pseaumes de Calvin ${ }^{3}$, en les complétant au besoin par les prescriptions littéraires des autres auteurs protestants qui relaient et amplifient la pensée du réformateur. À nous de décrypter les grandes lignes d'une poétique réformée et d'en mesurer la validité à l'aune des productions ultérieures des poètes. Mais pour entrer en propos, il nous faut d'abord replacer cette réflexion théorique dans le contexte littéraire du tournant du siècle.

\section{RUPTURES POÉTIQUES}

Avant le rappel à l'ordre des Réformés, certains poètes, comme Salmon Macrin, défendaient déjà, à l'orée du siècle, l'idéal d'une poésie chrétienne, incarnée par David, "prophète inspiré, expliquant les mystères sacrés sur sa lyre divine $»^{4}$. La condamnation de la Muse mondaine et la promotion érasmienne d'un nouveau modèle chrétien peinent cependant à entrer en résonance avec les pratiques des poètes humanistes de l'époque, adeptes des fictions païennes et enclins au lyrisme érotique. Il faut attendre la voix autoritaire d'un Calvin pour que résonne avec force en milieu réformé la condamnation de la poésie profane et que s'impose le modèle exclusif des Psaumes. Dans l'épître qui ouvre les éditions du Psautier huguenot à partir de 1543, le réformateur genevois rompt en visière

3. Nous nous appuierons surtout sur les Commentaires sur les Pseaumes, Genève, Conrad Badius, 1558, qui proposent les analyses les plus stimulantes sur les caractéristiques d'une poésie idéale, incarnée par la parole de David.

4. Cité par Jean Vignes dans Poétiques de la Renaissance, dir. Perrine GalandHallyn et Fernand Hallyn, Genève, Droz, 2001, chapitre IV (« Poésie et religion au XVI ${ }^{\mathrm{e}}$ siècle »), p. 265. 
avec les productions mondaines qui dénaturent, selon lui, l'origine et la fonction sacrées de la poésie :

Seulement que le monde soit bien advisé, qu'au lieu de chansons en partie vaines et frivoles, en partie sottes et lourdes, en partie sales et vilaines, et par consequent mauvaises et nuisibles, dont il a usé par cy devant, il s'accoustume ci apres à chanter ces divins et celestes Cantiques avec le bon Roy David .

Calvin solde l'héritage païen au nom de sa prétendue malfaisance morale, et lui substitue le modèle davidique, le seul qui soit légitime parce qu'il procède de Dieu et qu'il nous met en communication avec lui :

Parquoy quand nous aurons bien circui par tout pour cercher çà et là, nous ne trouvons meilleures chansons ne plus propres pour ce faire, que les Pseaumes de David, lesquels le sainct Esprit luy a dictez et faicts. Et pourtant, quand nous les chantons, nous sommes certains que Dieu nous met en la bouche les paroles, comme si luy mesme chantoit en nous, pour exalter sa gloire ${ }^{6}$.

Voilà l'exemple d'une belle réciprocité : l'Esprit fait retentir la harpe et la voix de David pour que le chant revienne à Dieu. Calvin met tous ses soins à remotiver l'articulation entre la louange et la lyre en insistant sur la consubstantialité du geste laudatif et de la parole poétique. Le don doit revenir à son donateur suivant un juste retour des choses. La polarité entre chant et célébration trouve son fondement dans le devoir de piété du poète chrétien, autorisé à user de sa plume, à charge de la mettre au service de Dieu qui l'inspire.

Le mot d'ordre de Calvin trouve un écho retentissant chez Marot ${ }^{7}$ et chez Bèze, lequel reprend à son compte en 1550, dans la préface de l'Abraham sacrifiant, les accusations du réformateur pour mener

5. Voir l'épître de Calvin en tête des éditions du Psautier huguenot à partir de 1543 ( « A tous chrestiens, et amateurs de la Parole de Dieu »), publiée par Gérard Defaux dans son édition des Cinquante Pseaumes de David de Clément Marot (Paris, Champion, 1995), p. 315-320.

6. Ibid., p. 319.

7. Clément Marot résume ces deux qualités dans l'épître au Roy qui ouvre sa traduction des Psaumes en 1543 (voir Cinquante Pseaumes de David, op. cit., p. 95-97, v. 39-80: «O donques Roy, prens l'euvre de David, / Euvre plustost de Dieu, qui le ravit, / D'autant que Dieu son Apollo estoit, / Qui luy en train et sa harpe mettoit, / Le saint Esprit estoit sa Caliope : / [...] Finalement son ruisseau cabalin / De Grace fut la fontaine profonde, / Où à grans traitz il but de la claire unde, / Dont il devint Poëte en un moment, / [...] / Icy sont donq les louanges escrites / Du Roy des Roys, du Dieu des exercites. / [...] / Icy oyt on l'Esprit de Dieu, qui crie / Dedans David, alors que David prie. » 
campagne contre le paganisme ostentatoire des premières œuvres de la Pléiade :

Que pleust à Dieu que tant de bons espriz que je cognoy en France, en lieu de s'amuser en ses malheureuses inventions ou imitations de fantaisies vaines et deshonnestes, (si on en veult juger à la verité) regardassent plustost à magnifier la bonté de ce grand Dieu, duquel ils ont receu tant de graces, qu'à flatter leurs idoles, c'est à dire leurs seigneurs ou leurs dames, qu'ils entretiennent en leurs vices, par leurs fictions et flatteries. A la verité il leur seroit mieux seant de chanter un cantique à Dieu, que de petrarquiser un Sonnet, et faire l'amoureux transy, digne d'avoir un chapperon à sonnettes : ou de contrefaire ces fureurs poëtiques à l'antique, pour distiller la gloire de ce monde, et immortaliser cestuy cy ou ceste là $[\ldots]^{8}$.

Les récriminations viennent après une palinodie qui deviendra monnaie courante dans la seconde moitié du siècle et surtout à partir des années 70, dans le contexte d'une conversion généralisée des Muses $^{9}$ :

Car je confesse que de mon naturel j'ay toujours pris plaisir à la poësie, et ne m'en puis encores repentir : mais bien ay-je regret d'avoir employé ce peu de grace que Dieu m'a donné en cest endroit, en choses desquelles la souvenance me fait maintenant rougir. Je me suis donques addonné à telles matieres plus sainctes, esperant de continuer cy apres : mesmement en la translation des Pseaumes ${ }^{10}$.

Revenu de ses erreurs passées, le poète peut désormais se poser en chef de file d'un renouveau de l'inspiration poétique dont les préceptes seront clairement énoncés en 1551 dans l'épître qui ouvre l'édition du Psautier huguenot: «Soyent desormais vos plumes adonnées / A louer Dieu, qui les vous a données $»^{11}$. Si Bèze renoue avec la transitivité idéale définie par Calvin et relayée par Marot, il insiste sur les modalités spirituelles d'une réforme poétique : «Pour ce faire, il faut premierement, / Que reformiez vos cœurs entierement. / Vos plumes lors d'un bon esprit poussees / Descouvriront vos divines pensées ${ }^{12}{ }^{12}$. Autrement dit, la conversion

8. Théodore de Bèze, Abraham sacrifiant, éd. Keith Cameron, Kathleen M. Hall et Francis Higman, Genève-Paris, Droz-Minard, 1967, p. 47.

9. On pourrait citer l'exemple de Joachim Du Bellay ou encore celui d'Agrippa d'Aubigné. Voir J. Vignes, op. cit., p. 264-271.

10. Bèze, Abraham sacrifiant, op. cit., p. 46.

11. Voir l'épître qui introduit les éditions du Psautier huguenot à partir de 1551, reproduite par Gérard Defaux dans son édition des Cinquante Pseaumes de David, op. cit., v. 127-128.

12. Ibid., v. 141-144. 
du cœur, voire de l'âme, est au principe d'une reviviscence du discours poétique. De toute évidence, Bèze place la christianisation de la Muse dans l'orbe pénitentielle: seule la régénérescence de l'être, subséquente au processus réparateur de la résipiscence, est en capacité de vivifier la langue poétique et de favoriser la consécration du poète: "Lors serez-vous Poëtes veritables $»^{13}$. Les écrivains reformés entérinent sans mal cette articulation originale entre poésie (de louange) et spiritualité (pénitentielle), en imputant leur conversion littéraire à la vivification de l'Esprit, accordée par faveur spéciale. L'ouverture des recueils met en scène cette transformation de l'âme qui induit la mue de la voix poétique, désormais portée à « ne chante[r] que de Dieu », pour reprendre l'expression d'Agrippa d'Aubigné ${ }^{14}$.

La réforme poétique passe donc, dans un premier temps, par un renouvellement du modèle (David) et par une révision du statut du poète (croyant vivifié par son engagement dans une démarche pénitente) avec, pour corollaire, une redéfinition de la fonction de la poésie : d'origine sacrée, celle-ci doit restreindre ses prérogatives à la gloire du Créateur. Ces principes posés, demeure l'essentiel : l'indication de normes concrètes pour l'élaboration d'une langue adéquate.

\section{PoÉSIE ET VÉRITÉ}

C'est la Bible qui fournit aux théoriciens du style réformé la matière d'une rénovation du langage poétique parce qu'elle leur permet notamment de résoudre l'articulation problématique entre art et vérité. À la suite d'Augustin (De doctrina christiana) et d'Érasme

13. Ibid., v. 145.

14. Agrippa d'Aubigné, Les Tragiques, éd. Jean-Raymond Fanlo, Paris, Champion, 1996, V, v. 1425. On se contentera de citer l'exemple éloquent d'Odet de La Noue dans ses Poesies chrestiennes, Genève, Vignon, 1594, « Cantiques chrestiens », 1, p. 87-88: "Touche mon ame au vif, afin qu'elle ressente / Les fautes qu'elle fait, et qu'elle s'en repente. / [...] / Et qu'est-ce qui me fait humilier ainsi, / Si ce n'est ta bonté qui mon courage touche? / N'est-ce pas toi, Seigneur, qui defermes ma bouche, / Pour me faire implorer ta divine merci? / Si ce n'est maintenant ton ardeur qui m'allume, / Qui fait tant regorger de saincts mots à ma plume? / C'est, c'est toi pour certain, c'est toi qui est l'autheur / De ce sainct changement dont mon ame est esprise. / [...] / Fay, fay que desormais on lise en mes escrits / Le veritable los de ta Majesté saincte, / Et que je laisse là mainte amoureuse feinte / Dont ma muse autrefois le sujet avoit pris / Et fai que sur mon luth à ton honneur je joue, / Et de voix et de cœur à jamais je te loue. » 
(Ecclesiastes), Calvin met en avant la dialectique paulinienne du message sublime et du style inculte pour prouver la supériorité absolue de la langue biblique : «[...] c'est la majesté de la matiere plus que la grace des parolles qui nous ravit en admiration d'icelle [...] $\gg^{15}$. Il fait appel au concept de la majestas, qui souligne l'infinie grandeur de Dieu, capable de rayonner et d'agir sur les âmes sans artifice rhétorique. Afin de justifier l'âpreté communément décriée de la parole sacrée, le théologien protestant avance l'argument émotionnel : l'Esprit touche les affects en suscitant une transformation radicale du cœur :

[...] telle simplicité rude, et quasi agreste, nous esmeut en plus grande reverence que tout le beau langage des Rhétoriciens du monde, que pouvons-nous estimer, sinon que l'Escriture contient en soy telle vertu de verité qu'elle n'a aucun besoing d'artifice de parolles? [...] Dont il est aisé d'apercevoir que les Sainctes Escritures ont quelque proprieté divine à inspirer les hommes, vues que de si loing elles surmontent toutes les grâces de l'industrie humaine. ${ }^{16}$

Calvin se livre du même coup à une critique de l'ornatus païen, inutile aux locuteurs bibliques, qui se veulent simples médiateurs du verbe divin. La rudesse de la langue est le gage d'une authenticité : les mots, réduits à des signes, n'ont d'autre fonction que d'amener l'âme à Dieu, seul objet de son amour. Loin de prétendre susciter un plaisir esthétique, ils ont charge de révéler les realia célestes sans truchement ornemental, et c'est précisément cette appréhension directe de la vérité qui génère l'émotion du lecteur. Contrairement à Augustin, Calvin renonce au delectare pour le movere, à condition qu'il soit dépouillé de tout art et qu'il fasse cause commune avec le docere. La simplicité de l'éloquence biblique constitue donc un

15. Jean Calvin, Institution de la religion chrétienne, éd. Jean-Daniel Benoît, Paris, Vrin, 1957-1963, I, 8, p. 101.

16. Ibid. Quelques décennies plus tard, D'Aubigné reprendra l'argument de Calvin pour défendre la supériorité du style biblique, désavoué par les traditions apostoliques autant que par les nouveaux poètes raffinés de la cour. Voir sa préface des méditations sur les Psaumes dans Petites Euvres meslees, éd. Véronique Ferrer, Paris, Champion, 2004 : « J'ay estimé estre à propos de faire voir comment parmi les styles les plus elabourés, [...] les passages de l'Escriture sont non seulement comme un esmail sur l'or, mais comme les pierreries exquises, et relevent le langage le plus eslevé; confirment par axiomes, preuvent par arrest du Ciel, illustrent par exemples, et recreent les esprits qui aiment Dieu par ravissantes lumieres et parfaites beautez. [...] mesme dans la rudesse de celle-là [la Vulgate] reluit tousjours la Majesté de celui qui prononce, et la richesse qui n'a besoin d'artifice, pour ravir à soi les yeux de l'ame et l'admiration des esprits. » 
modèle à double titre : d'abord, parce qu'elle nous met directement en contact avec la vérité, ensuite parce qu'elle touche immédiatement le cœur. En un mot, deux qualités propres à soutenir le projet d'édification qu'ambitionne la Réforme.

Dans la Vérité de la religion chrétienne (1581), Philippe DuplessisMornay, qui s'attarde assez longuement sur le style biblique en reconduisant les grands axes de la réflexion calvinienne, distingue tout particulièrement cette capacité exceptionnelle des Écritures à mettre les vérités les plus hautes à la portée des plus humbles :

Certes plus donq est simple la Loy, et mieux convient elle à l'Eternel; veu que plus simple elle est, et mieux represente elle la voix de celuy qui peut toutes choses : mais qui plus est, plus simple elle est, et mieux convient elle au peuple; car celle qui est ordonnee pour tous indifferemment, doibt estre comme une viande ordinaire; ou, pour mieux dire, comme un pain commun accommodé au goust et au palais de tous. ${ }^{17}$

La simplicité de la langue chemine de compagnie avec l'intelligibilité du sens que Calvin ne cesse de réclamer de lui-même et des autres : le prédicateur tout comme le poète doivent tendre vers la clarté pour aller au cœur de la pensée et du réel, pour la transmettre aussi aux autres. La complexité de la langue, qui porte à confusion la signification, constitue précisément l'un des griefs qu'adresse le réformateur aux Rhétoriciens ou aux libertins spirituels : «[...] ilz en deguisent tellement la signification, que jamais on ne sait quel est le subject de la matiere dont ils parlent $\gg{ }^{18}$. Parce que les contorsions du style relèvent d'une entreprise diabolique de dissimulation et de tromperie, elles contreviennent à l'enseignement de Dieu « qui use envers nous d'une maniere grossiere de parler, à fin d'estre entendu $»^{19}$. La sophistique est le contre-modèle de l'accomodatio, par laquelle Dieu témoigne à sa créature l'amour infini qu'il lui porte en se mettant à sa portée.

Le prédicateur comme le poète sont ainsi invités à se réapproprier la simplicité de la langue biblique en l'imitant, c'est-à-dire en délaissant les artifices de l'art oratoire au profit d'une éloquence

17. Philippe Duplessis-Mornay, De la verité de la religion chrestienne, Anvers, C. Plantin, 1581, p 612-618. Michel Jeanneret commente ces pages dans son ouvrage, op. cit., p. 168 sq.

18. Jean Calvin, Contre la secte phantastique et furieuse des Libertins qui se nomment spirituelz (1545), cité par M. Jeanneret, op. cit., p. 170.

19. Ibid. 
du cœur, en optant pour une syntaxe dépouillée de ses articulations complexes et en appauvrissant le lexique. Pour participer à cet ambitieux programme de vulgarisation et d'édification, les écrivains réformés renoncent aux finesses du métier. Mais, en tenant en lisière l'artifice littéraire, ils reprennent langue avec l'immédiateté de la parole sacrée; en mettant sous le boisseau les raffinements du style, ils renouent avec l'émotion primordiale du verbe divin. L'idéal de sincérité se fait au prix d'un effacement du poète, conformément du reste à l'humilité chrétienne. L'écrivain ne doit pas parler en son nom ni même mettre en avant son talent : sa tâche se borne à exprimer ce que Dieu lui dicte, soit à transmettre au plus près de son sens la vérité biblique. Si étroite soit leur marge de manœuvre, les poètes reformés ne rompent pas pour autant avec la littérature. En puisant dans des matériaux simples, ils renouvellent connaissance avec la langue française qu'ils font accéder à une autre forme de littérarité. Paradoxalement, ce qui pourrait apparaître comme une entreprise d'appauvrissement constitue à l'inverse une tentative d'enrichissement linguistique. Les poètes réformés introduisent dans la langue française des tours syntaxiques nouveaux, des hébraïsmes textuels, des mots tantôt concrets pour traduire le frémissement de la vie, tantôt abstraits pour décliner les affres du pécheur ou, à l'inverse, l'immense bonheur du croyant. Le style biblique fournit à ces auteurs les outils d'une exploration aiguë du réel le plus ordinaire et leur offre le large spectre des sensations, des sentiments et des émotions contradictoires de l'être. Il leur permet ainsi d'accueillir en littérature toute la complexité d'une réalité tangible, parfois rétive à la mise en mots, de même qu'il contribue à étoffer l'expression de leur intériorité, surtout de leur souffrance, déclinée en tous ses possibles sémantiques : affliction, géhenne, douleur, tourment, torture, etc. Sur ce chapitre, il n'est pas de concurrence pour le Psautier, qui constitue le modèle suprême d'une langue sensible à la variété des sentiments humains.

\section{LA LANGUE DU CEUR}

Depuis les Pères de l'Église, on s'accorde à décrire le livre psalmique comme une somme lyrique de toutes les Écritures, en plus de son aptitude à contenir leur enseignement théologique et moral. Si Basile et Athanase lui reconnaissent cette capacité à 
réfracter le savoir universel de la religion, ils font surtout ressortir sa vertu à réfléchir toute la complexité intérieure de 1 'homme ${ }^{20}$. Le Psautier engage donc tout autant une connaissance doctrinale qu'un savoir psychologique, en déclinant la palette de toutes les virtualités émotionnelles de l'être. De Luther à Calvin, les Réformés retiendront cette nouvelle lecture psychologisante des Psaumes au point d'élaborer à partir de leur commentaire une véritable dramaturgie universelle des passions humaines. Si Calvin insiste, dans la célèbre préface de ses Commentaires sur les Pseaumes, sur l'enseignement doctrinal du livre davidique, véritable «thresor» d'une «merveilleuse utilité », grâce auquel le chrétien comprendra « la plus grand'part de la doctrine celeste $»^{21}$, il s'attarde plus volontiers sur son efficacité spéculaire, qui l'autorise à témoigner de l'expérience singulière du croyant, en vertu d'un principe d'identification direct du lecteur contemporain avec le personnage biblique. Plus que tout autre livre, le Psautier, qui met à nu le cœur de l'homme en dépeignant la confusion des sentiments et l'agitation des émotions, se prête à une lecture introspective :

J'ay accoustumé de nommer ce livre une anatomie de toutes les parties de l'ame, pource qu'il n'y a affection en l'homme laquelle ne soit icy representee comme en un miroir. Mesme, pour mieux dire, le S. esprit a yci pourtrait au vif toutes les douleurs, tristesses, craintes, doutes, esperances, sollicitudes, perplexitez, voire jusques aux esmotions confuses desquelles les esprits des hommes ont accoustumé d'estre agite ${ }^{22}$.

Pareille interprétation infléchit profondément la méthode exégétique de Calvin. Commenter le Psautier revient moins à dégager le contenu doctrinal du texte ou à lui appliquer une lecture théologique, qu'à apprécier la sphère de pertinence de son message au regard de l'expérience commune du fidèle, bref à mesurer l'adaptabilité de la parole davidique au nom de la propriété intersubjective qui la caractérise. Dans sa préface, Calvin se prévaut d'une coïncidence existentielle entre David et lui-même, qu'il démontre par

20. Voir notamment l'Epistola ad Marcellinum de saint Athanase. Sur l'influence de cette lecture des Psaumes sur Luther et Calvin, voir Barbara K. Lewalski, Protestant Poetics and the Seventeeth-Century Religious Lyric, Princeton University Press, Princeton, New Jersey, 1979, chapitre 2.

21. Jean Calvin, Commentaires sur les Pseaumes, op. cit., Préface non paginée.

22. Ibid. 
une longue mise en scène autobiographique, à dessein de légitimer implicitement sa clairvoyance à l'égard du Psautier :

[...] ce m'a esté une grande consolation de me conformerà l'exemple d'un tel personnage et si excellent. Et mesmes ceste cognoissance et experience m'a de beaucoup servy pour entendre les Pseaumes, affin que je ne m'y trouvasse trop nouveau comme en un pays incognu. Et de faict, les lecteurs (comme je pense) recognoistront qu'en declairant les affections interieures tant de David que des autres, $j$ 'en parle comme de choses desquelles j'ay familiere cognoissance ${ }^{23}$.

Tenu pour double de David, l'exégète apparaît donc tout désigné pour percer à jour le versant secret de sa parole. Fort de cette convergence affective, il peut sans mal accéder aux tréfonds du cœur du psalmiste et donner une cohérence aussi bien spirituelle que théologique aux excès de ses passions. L'application de sa nouvelle méthode exégétique, fondée sur une lecture littérale des textes bibliques, conduit Calvin à s'attacher à la littérarité de la parole davidique. En effet, renonçant à l'opposition traditionnelle entre sens littéral et sens spirituel, il se propose, à travers la mise en contexte de «chaque élément sémantique et discursif $»^{24}$ ainsi qu'à travers l'analyse philologique et rhétorique des intentions de l'auteur, de mettre en évidence les versants moral et théologique du sens littéral. Autant dire que, dans le genre lyrique des Psaumes, cette méthode l'amène en bonne logique à commenter le lexique affectif du psalmiste aussi bien que les multiples figures dont sa prière à Dieu se nourrit. La lecture rhétorique de l'exégète ouvre la voie à une lecture littéraire non avouée de l'oraison, qui fournit des indications précieuses sur l'idéal poétique de Calvin. En marge du commentaire théologique s'élabore donc un art de la prière, à destination du poète autant qu'à celle du commun des fidèles.

C'est que la poésie ne saurait s'envisager hors de l'orbe d'une spiritualité privée : l'exercice poétique se confond avec l'élan de la prière qu'il doit élever de la singularité jusqu'à l'exemplarité à la façon du psaume. Le poème n'est rien d'autre finalement que la textualisation de l'oraison : il fait accéder la parole souterraine et confuse du croyant à l'expression articulée de ce qui, sans le processus poétique régulé par l'imitation psalmique, ne serait que cris et

23. Ibid.

24. Voir Olivier Millet, Calvin et la dynamique de la parole, Etude de la rhétorique réformée, Paris, Champion, 1992 p. 289. 
gémissements; il hisse aussi au rang de l'écriture certains moments fondamentaux de l'expérience spirituelle. À la différence de la théologie, la poésie préserve le domaine religieux du danger spéculatif. Les interrogations que soulève le croyant restent incluses dans les bornes de l'adresse à Dieu, elles ne s'émancipent pas du cadre empirique et se maintiennent dans les limites existentielles. Dans le même temps, la poétisation de la prière favorise l'expression individuelle d'une spiritualité universelle, le chant unique d'une foi partagée. La translation poétique décontextualise l'expérience unique du psalmiste et la recontextualise par un réinvestissement affectif autant que par une réappropriation linguistique. Ce jeu entre singularisation et généralisation marque l'avènement d'un lyrisme intersubjectif, qui permet par la « délocalisation » féconde du sujet d'en colmater les fissures profondes.

Si elle est à l'origine d'un lyrisme original, l'exclusivité du modèle littéraire - la prière psalmique - circonscrit le sujet et l'énonciation poétiques. Pour cette dernière, deux choix s'offrent au poète : la supplique et la louange, à savoir les deux versants souvent conjoints de la prière davidique. Le psaume décline une parole moins réflexive que transitive, rythmée par le vocatif de la supplique et de la célébration, de la plainte et de l'action de grâces. $\mathrm{Si}$, pour Calvin, la glorification divine prend rang dans la hiérarchie très stricte des exercices de piété avant tous les autres ${ }^{25}$, afin d'être efficace et authentique elle doit épouser l'élan d'humilité qui préside à l'invocation - « le vray sacrifice de foy $»^{26}-$, soit s'intégrer à la dynamique de la pénitence. D'où la valorisation spirituelle et métapoétique de la supplique par le commentaire calvinien au détriment de la louange, valorisation que les poètes vérifieront dans leur pratique littéraire. La lecture pénitentielle du Psautier par le réformateur a pour conséquence de borner le sujet poétique à l'itinéraire spirituel du fidèle, qui le mène de la confession des fautes (« mortification de la chair ») jusqu'à la régénérescence (« vivification de l'Esprit »). La prière, selon Calvin, actionne les mécanismes d'une analyse autocritique qui révèle l'être à lui-même, c'està-dire à sa nature corrompue. Elle prend la forme d'une confession

25. «C'est le principal sacrifice, que le sacrifice de louange et aussi c'est le vray tesmoignage de pieté », Commentaires sur les Pseaumes, op. cit., p. 415.

26. Ibid., p. 202. 
qui équivaut in fine à une mise à plat de la corruption originelle de l'homme : « [...] la connaissance d'un peché, commente Calvin, amène David à faire un long examen de toute sa vie jusques là qu'il ne trouve rien en soy que peché $[. .$.$] il se confesse une nature$ corrompue et damnable $»^{27}$. L'autodénigrement et l'humiliation qui l'accompagne sont les conditions requises pour l'obtention du salut: «[...] nul ne peut avoir esperance de salut, sinon qu'il se vienne humilier et prosterner devant Dieu ${ }^{28}$. Encore faut-il que l'humiliation de soi se double d'un appel à la gratuité de la miséricorde divine : «David recourant aux misericordes [...] de Dieu, rend tesmoignage qu'il ne presume rien de ses merites, et n'y met aucune esperance. Car celuy qui puise tout de la seule misericorde de Dieu, c'est bien à dire qu'il ne trouve rien en sa propre personne qui merite recompense devant Dieu $»^{29}$. L'appel à la miséricorde de Dieu traduit un anéantissement devant la puissance céleste, et constitue l'aveu d'une nullité consubstantielle, interprétée comme une conséquence de la corruption originelle.

L'interprétation calvinienne de la prière davidique informe les cadres d'un nouveau lyrisme, un lyrisme négatif, où le sujet se dévoile pour mieux se nier, se révèle pour mieux s'anéantir. La mise à nu du cœur, loin d'une exhibition complaisante des sentiments, prend le tour d'un procès sans ménagement de l'être. L'expression de soi est une condamnation de soi. Ce pessimisme ontologique trouve à s'exprimer dans le réalisme cru des images bibliques, qui dressent le portrait d'un être contaminé par l'ordure, voué à la pourriture, rivé à la misère terrestre sans le secours divin. La radicalité du verbe trouve cependant une efficacité dans la dynamique de la pénitence. «Cet amas de parolles, dit Calvin à propos du Psaume 51, montre combien [David] estoit soucieux d'impetrer pardon ». « [...] le pecheur tant plus il se sent souillé, d'autant plus se doit il inciter à gemissemens continuels et affectueux, afin de venir finalement au dessus des espouvantemens de la conscience $»^{30}$. L'excès à la fois sémantique et quantitatif du langage répond donc à une nécessité spirituelle : ce n'est que par une plainte assidue et ardente que le fidèle peut à la fois exorciser sa monstruosité et forcer l'écoute

27. Ibid., p. 343-344.

28. Ibid., p. 202.

29. Ibid., p. 153.

30. Ibid., p. 340-341. 
de Dieu. L'hypertrophie de la confession apparaît comme une condition préalable à l'obtention du salut, la dissolution de l'être comme une préparation à la réconciliation qui s'accomplira au moment de la régénération spirituelle de la pénitence. Cette métamorphose se traduit sur le plan intratextuel par un renversement énonciatif de la plainte en louange, d'un verbe sans espoir en une parole de confiance, d'un lyrisme de l'affliction en un lyrisme de l'enthousiasme. À la transformation spirituelle correspond donc une mutation poétique qui relève d'une dramatisation concertée de l'intériorité du croyant et de la langue du poète, d'une exhibition des passions autant que d'une exaltation des mots.

Le commentaire calvinien s'attache comme nul autre au détail de la langue davidique dans laquelle le réformateur voit le théâtre expressionniste des affections humaines. Il met l'accent sur la véhémence du style psalmique, où se multiplient les figures de l'hyperbole, de l'accumulation et de la gradation. La démesure verbale n'a pourtant rien d'une exagération mensongère, elle constitue au contraire l'exacte traduction du sentiment outré qu'éprouve le fidèle :

Combien que ces façons de penser soyent hyperboliques, c'està-dire comme excessives et passant mesure, touttefois David n'amplifie pas la tristesse à la façon des Poëtes, mais declare vrayement et simplement combien elle est dure et aspre. ${ }^{31}$

La langue hyperbolique ne présente pas un excès du signifiant par rapport au signifié comme chez les poètes - ceux que Calvin condamne parce qu'ils privilégient l'ornement au détriment du sens -, elle est une réponse motivée à une affectivité surtendue, elle est à proportion de la souffrance ressentie. " David n'emprunte point une rhetorique fardee $»^{32}$, car l'outrance ne ressortit pas chez lui à l'artifice du verbe mais à la vérité des émotions :

[David] monstre la cause pourquoy il insiste si affectueusement et ardemment à demander pardon, ascavoir parce qu'il ne peut demeurer en repos, jusques à ce qu'il soit reconcilié avec Dieu. Et ainsi il rend temoignage qu'il ne prononce pas un mot par feintise : comme aucuneffois nous en voyons qui exaltent par un amas de termes hauts et magnifiques la grace de Dieu touchant la remission des pechez, lesquels touttefois, y pesent bien maigrement, d'autant qu'ils ne sont gueres tou-

31. Ibid., p. 340-341.

32. Ibid., p. 253. 
chez du sentiment de son jugement. David donc afin d'obtenir pardon ne dit pas sans cause qu'il est agité de tourments continuels, et que l'inquietude laquelle il sent le contraint à prieres ainsi ardentes. ${ }^{33}$

Réduite à la clameur stérile, enfermée dans la déploration ressassante, ouverte à l'invocation dynamique ou à la célébration enthousiaste, la parole davidique offre, selon Calvin, l'exemple d'une voix authentique, en adéquation parfaite avec les sentiments qu'elle a en charge de transmettre, le modèle d'une langue affective qui actualise l'exigence divine d'une coïncidence absolue entre la « bouche « et le « cœur». Or, cette coïncidence n'est possible que dans l'élan impérieux de la prière, qui débarrasse la parole des contraintes de l'esprit et des artifices de la rhétorique. Dans cette situation d'urgence et de désespoir s'élabore une langue immédiate et pure, une langue entière et émotionnelle, indifférente aux normes de la raison : «[...] c'est l'office du cœur d'esmouvoir la langue et de la bien disposer à prier [...] Il y a en ceci quelque chose de reciproque et mutuel : car comme le cour doit dicter et former les parolles, ainsi la langue aide à la parolle du cœur $»^{34}$. Tout en décrivant les caractéristiques du style davidique, Calvin fustige en sousroche l'affectation et l'enjolivement, la mièvrerie et le maniérisme de la poésie contemporaine, dont il déplore surtout l'inauthenticité. Il élabore une poétique de la prière qui prend à rebours les critères esthétiques en vigueur : véhémence spontanée et simplicité agreste concourent à ériger le modèle d'une langue antipoétique, dont la puissance résiderait principalement dans sa charge émotionnelle, une langue, dira plus tard Aubigné, « qui n'a besoin d'artifice, pour ravir à soi les yeux de l'ame et l'admiration des esprits $»^{35}$.

De la glose exégétique au manuel de piété, du traité de prière au manifeste poétique, le commentaire calvinien connaît des glissements génériques qui s'expliquent par la jonction dynamique entre connaissance, foi et langage. Connaître Dieu, c'est l'invoquer; prier, c'est d'abord trouver une langue audible et efficace. Le Psautier offre au réformateur l'exemple de l'articulation féconde entre poésie et spiritualité, propre à ressourcer selon lui la poésie contemporaine, dévoyée par la fable et l'ornement. Dans son commentaire, il justifie

33. Ibid., p. 341.

34. Ibid., p. 63.

35. Aubigné, Petites Euvres meslees, op. cit., Préface. 
par le menu le modèle qu'il brandissait sans plus d'arguments dans ses premières épîtres. Il trace les grandes lignes d'une poétique des affections qui se propose tacitement de remettre à neuf les critères linguistiques de la littérature de son temps et de rénover les fondements du lyrisme sous le surplomb de la foi. Cette réforme qu'il mène discrètement sous l'autorité du théologien exclut pourtant toute réflexion formelle : nulle indication sur la prosodie ni sur les genres poétiques. Et pour cause : Calvin n'est ni poète ni poéticien de métier; son aversion pour l'artifice ne peut l'incliner qu'en faveur du psaume, à l'identité formelle des plus souples. Il n'empêche : la poésie exige dans une littérature extrêmement codifiée un moule contraignant où se couler. Le silence calvinien aurait pu être comblé par un poète réformé. Ce ne fut pas le cas. Cette lacune ou plutôt cette absence de directives limitera l'étendue de l'entreprise menée en solitaire par le réformateur. C'est précisément à mesurer la portée réelle de la réforme calvinienne chez les poètes, ses éclats comme ses échecs, qu'il convient désormais d'appliquer la réflexion.

\section{À L'ÉPREUVE dES FAITS}

Les appels de Calvin et de Bèze logés dans les premières éditions du Psautier huguenot renversent la perspective poétique en milieu réformé. Si les poètes de la cour peinent encore, face à la vogue du pétrarquisme et aux règlementations de la Pléiade, à opter pour une lyre exclusivement chrétienne, les poètes protestants se lancent à corps perdu dans une production religieuse pour l'essentiel polémique, doctrinale ou morale ${ }^{36}$, conformément aux ambitions d'édification de la Réforme. Reste que le versant lyrique de l'inspiration religieuse, celui qui nous intéresse ici, compte moins de représentants, constat imputable sans doute à l'autorité du Psautier huguenot, lequel, tenu pour une somme, réalise finalement le vœu poétique des réformés, et décourage longtemps toute autre entreprise d'envergure analogue. Certains poètes s'y risquent néanmoins en composant des paraphrases de psaumes dès les années 50 comme

36. Voir Jacques Pineaux, La Poésie des protestants de langue française (1559-1598), Paris, Klincksieck, 1971. 
Guillaume Guéroult et Bruno Des Masures ${ }^{37}$, répondant à leur manière à l'idéal calvinien d'une poésie d'inspiration davidique. Mais il faut attendre les années 70 et 80 pour que se généralise ce courant d'écriture, moins sous le nom de paraphrases que sous l'appellation de cantiques spirituels, de chansons, d'imitations, de consolations, de méditations ou tout simplement de poésies chrétiennes. Ce sont là autant de titres qui déclinent le plus souvent un seul et même genre : la prière pénitente, suppliante, désespérée, confiante ou laudative du fidèle. Quelle que soit la forme choisie, le poème rejoue sans fin le drame intérieur du chrétien rongé par ses doutes, bourrelé de remords, ravagé par la souffrance, accablé par la déréliction, apaisé par la foi, revigoré par l'espérance. Des Chansons spirituelles à l'honneur et louange de Dieu (1569) aux cantiques de Maisonfleur ou à ceux de Valagre (1581), des Imitations chrestiennes de Simon Goulart (1574) aux Poesies chrestiennes d'Odet de La Noue (1594), des prières de La Calliope chrestienne de Benoît Alizet (1593) à celles d'André Mage de Fiefmelin (1601) ou à celles de L'Hyver d'Agrippa d'Aubigné, publiées à la veille de sa mort en 1629 , le poème reconduit l'interlocution problématique de la prière psalmique de même qu'il renoue avec le théâtre exacerbé des affections. Sans le déclarer ouvertement, l'oraison davidique dans sa diversité constitue le modèle unique de cette poésie à qui elle fournit un mode d'énonciation, une langue, un cadre spirituel, une thématique ainsi qu'un arsenal d'images motivées. Sans sombrer dans la veine doctrinale, le poème réinterprète néanmoins la prière psalmique à l'aune de la lecture calviniste. Le croyant, le véritable sujet lyrique, fait, dans l'espace circonscrit de l'oraison, l'expérience intérieure de la parole divine et de sa puissance de transformation. La prière réformée actualise dans le verbe et dans le cœur le dogme de la justification par la foi : elle avalise au fond de l'âme la doctrine du salut. Du coup, elle donne tout son sens au renversement énigmatique de la supplique en louange, du désespoir en espoir, que les poètes dramatisent par une rupture rythmique. D'un état à l'autre se joue dans le blanc du texte l'action miraculeuse de Dieu, l'illumination de l'âme du fidèle par l'Esprit Saint.

37. Voir respectivement Guillaume Guéroult, Premier livre des Pseaumes, Cantiques et Chansons spirituelles, Genève, 1554, et Bruno Des Masures, Vingt pseaumes de David traduits selon la verité hebraique et mis en rime françoise, Lyon, J. de Tournes et G. Gazeau, 1557. 
Si les poètes réformés ratifient par une pratique unanime la spiritualisation du langage poétique que Calvin appelle de ses vœux, ils divergent sur les suites à donner aux moyens littéraires proposés par le réformateur et par ses disciples. À commencer par l'imitation de la simplicité agreste de la langue biblique, nécessaire à un ressourcement authentique et émotionnel du verbe poétique. Pour l'essentiel, les poètes donnent leur aval au dépouillement formel prôné par Genève, ainsi qu'à l'influence hébraïque, au premier chef Marot et Bèze, qui se distinguent par la rigueur de leur version du Psautier. Ils tentent de créer, chacun à sa manière, une poésie dense et claire, concrète et accessible, débarrassée des ornements de la rhétorique et des enjolivements païens. Le genre de la paraphrase favorise tout particulièrement le mimétisme biblique : syntaxe, lexique et figures sont marqués au chiffre de la langue sacrée. Certains poètes poussent les feux de ce processus de rénovation en radicalisant l'autorité du modèle biblique. Aubigné est de ceux-là, qui fait de la rudesse sa marque de fabrique poétique au nom de la vérité qui lui est afférente. La langue de Canaan, dont il se réclame, est une langue anti-poétique selon les critères du temps, déplaisante, abrupte et qui assume pleinement son imperfection. À l'image de la parole sacrée, elle perd en élégance ce qu'elle gagne en puissance émotionnelle. Aubigné s'aligne sur l'argument calvinien de l'émotion pour mener son plaidoyer en acte, lui qui prend précisément à tâche d'émouvoir (movere), non d'enseigner (docere), encore moins de plaire (delectare) ${ }^{38}$. Dans le genre lyrique de la poésie réformée, Aubigné constitue à coup sûr une exception : il est, sur le plan poétique, sinon doctrinal, le plus authentiquement calviniste, même s'il n'échappe pas, surtout dans Les Tragiques, à un syncrétisme nécessaire. Il porte à son accomplissement le rêve d'une langue vraie et efficace, même s'il doit, pour ce faire, aller à l'encontre de certaines prescriptions. Reste que, dans le même temps, il est l'ultime représentant - un représentant anachronique au début du XVII ${ }^{\mathrm{e}}$ siècle - de ce courant poético-spirituel lancé par Calvin.

La radicalité d'une telle entreprise rencontre peu d'adeptes. La poésie lyrique, à la différence de la poésie polémique qui trouve dans la langue de Canaan une identité efficace, se montre peu encline à rompre le cercle fatal de l'héritage païen et à se défaire de l'ascendant

38. Voir A. d'Aubigné, LesTragiques, “Aux lecteurs ”, éd. cit., p. 13. 
poétique de La Pléiade. Contrairement aux catholiques, qui officialisent d'une certaine manière le syncrétisme religieux, les réformés restent internes à la doctrine selon laquelle la fable antique, si prestigieuse soit-elle, dénature la poésie religieuse. Il faut donc l'éradiquer sous peine d'attenter à la pureté du langage spirituel. Rien de plus difficile cependant à en lire la plupart des grands poètes de la Réforme, qui tiennent le milieu entre imitation biblique et inspiration antique : de Du Bartas à d'Aubigné, pourtant ardent défenseur du style des Écritures et représentant majeur de la rudesse poétique, en passant par Sponde, Poupo ${ }^{39}$ ou même Goulart, tous sont partagés entre les directives calviniennes et l'influence décisive de la Pléiade. Ils ont beau tenir sous surveillance étroite les critères novateurs de celle-ci, ils en reconnaissent tacitement l'autorité en adaptant son enseignement linguistique et littéraire à leurs préoccupations spirituelles, en reconduisant aussi les formes poétiques qu'elle valorise : sonnet, ode, élégie, épitaphe. Le sonnet et l'ode s'avancent en surplomb dans la hiérarchie des genres prisés par les écrivains réformés ${ }^{40}$. Ils concurrencent nettement le psaume, l'hymne ou le cantique, qui se déclinent souvent sous la forme de vers suivis ou de strophes de longueur variable (quatre à huit vers) avec alternance rimique. Les innovations formelles des poètes restent donc limitées faute d'une réflexion théorique aboutie, faute aussi d'une nette démarcation avec les écrivains de la Pléiade, qui demeurent, au-delà des critiques lancées, des maîtres incontestés.

Sans faire de la virtuosité du style ni de la recherche lexicale la marque distinctive du bon poète comme les théoriciens de La Pléiade, les poètes réformés valorisent la beauté objective de la forme. Si la parole psalmique reste un modèle de prière et d'écriture, elle doit compter avec l'ingéniosité verbale du poète. Les vers d'un Sponde ou d'un Du Bartas trahissent un attachement tout littéraire à la matière sémantique et sonore des mots, qui résiste à la stricte instrumentalisation

39. Voir l'introduction d'Anne Mantero dans son édition de La Muse chrétienne, Société des Textes Français modernes, 1997, p. XXXI.

40. Dans la plupart des recueils religieux, la prière se coule dans le moule du sonnet et de l'ode : ils constituent l'essentiel des Imitations chrestiennes de Goulart, des Poesies chrestiennes de La Noue. Bèze compose une prière de demande sous forme d'ode, "Ode chantee au Seigneur » dans Poemes chrestiens et moraux, sl nd. Voir aussi les poèmes chrétiens de Montméja (Genève, Jacob Stoer, 1574), les odes chrétiennes de Jean Tagaut (id), les sonnets de L'Exercice spirituel de Paul Perrot de la Salle (Saumur, Thomas Portau, 1606), etc. 
chrétienne des verba. La magnificence de la langue tend à attirer les regards sur elle au détriment du sujet et, de fait, à écorner le pacte poétique de transparence absolue. Le vœu de neutralité linguistique et d'effacement identitaire reste un idéal, que la mise en forme littéraire compromet à coup sûr. À l'heure d'écrire, le poète laisse la plume aller à discrétion : la personnalité de l'écrivain reprend ses droits sur l'humilité du fidèle, les acquis d'un long métier le disputent aux devoirs de piété. Le langage poétique tient lieu de contre-lettre modifiant le contrat spirituel et pastoral du poète chrétien, sans pour autant dénaturer sa foi.

La réforme poétique menée par Calvin et ses disciples au tournant du siècle eut un retentissement aussi éclatant qu'éphémère. Elle fraya les voies à une poésie originale, éprise de spiritualité et d'authenticité, en la retrempant aux sources vives des Écritures; elle contribua à remotiver le lyrisme en reprenant langue avec les émotions les plus vives du croyant; elle forgea un nouvel idiome propre à exprimer les modulations d'une foi tourmentée. Cette entreprise ambitieuse achoppa cependant aux règles internes de l'œuvre littéraire et souffrit de la difficile jonction entre religion et poésie, qui devient presque improbable sous le regard sourcilleux d'un Calvin. À la vision rapprochée, sa théorie poétique se fonde paradoxalement sur une négation de la poésie dans ce qui la définit en tant que forme littéraire : en proscrivant l'artifice en tout genre, en désavouant la contrainte formelle, elle la frappe dans ses œuvres vives. Elle exige finalement de la poésie qu'elle sacrifie une part d'elle-même sur l'autel de la religion. Un tel sacrifice est loin de rallier tous les suffrages, comme le prouvent les entorses presque systématiques à la loi calvinienne, y compris chez des poètes/théologiens comme Bèze. À tant bâillonner l'expression poétique, à tant la « dépoétiser » au nom de la vérité chrétienne, les réformateurs ont réduit malgré eux le cercle de leurs adeptes, ou à tout le moins ont limité la sphère d'influence de leur courant sur la littérature. Si les poètes adhèrent sans restriction aux préceptes spirituels de la poétique calvinienne, ils ont plus de difficulté à suivre son programme formel. Cette réserve explique sans doute que la réforme littéraire n'ait pas résisté au pressoir du temps ni à l'affaiblissement du protestantisme en France. Après la promulgation de l'Edit de Nantes, voire avant même sa promulgation, les poètes réformés prennent du champ par rapport au programme calvinien dans 
lequel ils ne se reconnaissent plus et dans lequel, de manière plus prosaïque, ils ne trouvent plus leur compte. À l'heure où triomphent dans les salons l'élégance et la mesure, où s'impose le modèle spirituel et rhétorique de l'éloquence dévote, où s'émoussent dans la poésie les marquages confessionnels ${ }^{41}$, la parole radicale et intransigeante d'un Calvin se perd, couverte par les voix d'un autre renouveau.

veronique-ferrer@orange.fr

41. Voir l'article de Julien Gœury dans ce même numéro. 